\title{
The oncolytic effects of reovirus in canine solid tumor cell lines
}

\author{
Masaya IGASE ${ }^{1)}$, Chung Chew HWANG ${ }^{2}$, Matt COFFEY ${ }^{3)}$, Masaru OKUDA ${ }^{4,5)}$, Shunsuke NOGUCHI ${ }^{1,2,5)}$ and \\ Takuya MIZUNO ${ }^{1,2,5) *}$ \\ 1)Laboratory of Molecular Diagnostics and Therapeutics, Joint Faculty of Veterinary Medicine, Yamaguchi University, Yamaguchi \\ 753-8515, Japan \\ ${ }^{2)}$ Laboratory of Molecular Diagnostics and Therapeutics, The United Graduate School of Veterinary Science, Yamaguchi University, \\ Yamaguchi 753-8515, Japan \\ 3) Oncolytics Biotech Inc., Calgary, Alberta, Canada \\ ${ }^{4)}$ Laboratory of Veterinary Internal Medicine, Joint Faculty of Veterinary Medicine, Yamaguchi University, Yamaguchi 753-8515, Japan \\ ${ }^{5)}$ Biomedical Science Center for Translational Research, The United Graduate School of Veterinary Science, Yamaguchi University, \\ Yamaguchi 753-8515, Japan
}

(Received 29 October 2014/Accepted 2 January 2015/Published online in J-STAGE 16 January 2015)

ABSTRACT. Oncolytic virotherapy is a new strategy for cancer treatment for humans and dogs. Reovirus has been proven to be a potent oncolytic virus in human medicine. Our laboratory has previously reported that canine mast cell tumor and canine lymphoma were susceptible to reovirus. In this study, canine solid tumor cell lines (mammary gland tumor, osteosarcoma and malignant melanoma) were tested to determine their susceptibility towards reovirus. We demonstrated that reovirus induces more than $50 \%$ cell death in three canine mammary gland tumors and one canine malignant melanoma cell line. The reovirus-induced cell death occurred via the activation of caspase 3 . Ras activation has been shown to be one of the important mechanisms of reovirus-susceptibility in human cancers. However, Ras activation was not related to the reovirus-susceptibility in canine solid tumor cell lines, which was similar to reports in canine mast cell tumor and canine lymphoma. The results of this study highly suggest that canine mammary gland tumor and canine malignant melanoma are also potential candidates for reovirus therapy in veterinary oncology.

KEY WORDS: cancer, canine, oncolytic virotherapy, reovirus, solid tumor

doi: 10.1292/jvms.14-0570; J. Vet. Med. Sci. 77(5): 541-548, 2015

Cancer is the leading cause of death in dogs $[7,16]$. The most common tumor in dogs is skin tumor [12, 16], but other malignant tumors, such as lymphoma, mammary gland tumor (MGT), osteosarcoma and malignant melanoma, are also commonly diagnosed and attribute to the cause of death. Due to the aggressive characteristic of this disease and its refractory to conventional therapy, there is a dire need for a new therapy that fights against cancer more effectively. Recently, various new therapeutic approaches have been developed in human oncology, and these new cancer therapies were also used for pet animals.

Oncolytic virotherapy is one of the latest strategies for cancer therapy. Basically, oncolytic viruses infect and kill tumor cells without affecting normal cells. Replication of these viruses will take place in the infected tumor cells, leading to cell lysis and release of viruses, which will again attack other tumor cells. Many viruses have been developed for oncolytic virotherapy, and some of them are currently in human clinical trials in various parts of the world [30]. Most of the oncolytic viruses are genetically modified to make them tumor specific

\footnotetext{
*Correspondence to: Mizuno, T., Laboratory of Molecular Diagnostics and Therapeutics, Joint Faculty of Veterinary Medicine, Yamaguchi University, 1677-1 Yoshida, Yamaguchi 753-8515, Japan. e-mail: mizutaku@yamaguchi-u.ac.jp

(C)2015 The Japanese Society of Veterinary Science

This is an open-access article distributed under the terms of the Creative Commons Attribution Non-Commercial No Derivatives (by-nc-nd) License $<$ http://creativecommons.org/licenses/by-nc-nd/3.0/>.
}

and to reduce the pathogenicity of the viruses. Using genetically modified oncolytic virus in a clinical setting in small animal medicine is unrealistic, because of the difficulty in management of the patients treated with the virus and the subsequent viral excretion. On the other hand, some viruses including reovirus and myxoma virus have been discovered to be naturally occurring oncolytic viruses without any genemodifications [10, 13, 29].

Reovirus is a member of the Reoviridae family and was isolated from the human respiratory and gastrointestinal tracts [20]. As a non-enveloped double-stranded RNA virus, pathogenicity of reovirus is low where most adults are seropositivity albeit not showing any clinical symptoms $[22,26]$. Despite the lack of pathogenicity in humans, reovirus has shown selective infection in transformed or malignant tumor cells with a preference to replicate in these cells $[5,8]$. Oncogenic Ras-transformed cells are highly susceptible to reovirus infection, and non-transformed cells did not allow the translation of viral genes and viral replication. The major difference between reovirus susceptible and non-susceptible cells lies in the ability of the cells to phosphorylate PKR (dsRNA-activated protein kinase). In cases of reovirus infection in susceptible cells, Ras activation will inhibit the phosphorylation of PKR and allow the expression of viral proteins before the release of viral progeny. However, it has also been reported that reovirus can exert oncolysis independent of this pathway in some tumor cells [28].

Our laboratory has previously reported that canine mast cell tumor was highly susceptible to reovirus and is a poten- 
tial candidate for oncolytic virotherapy using reovirus [10]. We also discovered that four out of ten canine lymphoma cell lines were susceptible to reovirus [9]. In order to find out if oncolytic virotherapy using reovirus is feasible in canine solid tumors, we tested the susceptibility of reovirus in canine MGT, osteosarcoma and malignant melanoma cell lines in this study. We also examined the relationship between Ras activation status and reovirus susceptibility in all the cell lines.

\section{MATERIALS AND METHODS}

Cell lines and reovirus: Seven established canine MGT cell lines (CIP-p, CIP-m, CTB-p, CTB-m, CHM-p, CHM$\mathrm{m}$ and CNM-m [17]), 5 canine osteosarcoma cell lines (Abrams, D17, Gracie, MacKinley and Moresco [14]) and six canine malignant melanoma cell lines $(\mathrm{CMeC} 1, \mathrm{CMeC} 2$, $\mathrm{KMeC}$, LMeC [11], CMGD2 and CMGD5 [2]) were used in this study. All canine MGT and 4 canine malignant melanoma cell lines (CMeC1, $\mathrm{CMeC} 2, \mathrm{KMeC}$ and $\mathrm{LMeC}$ ) were kindly provided by Dr. Nakagawa. Two canine malignant melanoma (CMGD2 and CMGD5) and all canine osteosarcoma cell lines were kindly provided by Dr. Modiano and Dr. Thamm, respectively. Mouse L929 fibroblastic cell line was obtained from the Cell Resource Center for Biomedical Research (Institute of Development, Aging and Cancer, Tohoku University, Sendai, Japan) and was used in the titration of viral progeny. The human Burkitt's lymphoma cell line, Raji, which was used as a positive control in the Ras GST pull-down assay, was obtained from the Cell Resource Center for Biomedical Research. All cell lines were grown in the R10 complete medium (RPMI1640 supplemented with $10 \% \mathrm{FBS}, 100 \mathrm{U} / \mathrm{m} l$ penicillin, $100 \mu \mathrm{g} / \mathrm{m} l$ streptomycin and $55 \mu \mathrm{M} 2$-mercaptoethanol) and were maintained at $37^{\circ} \mathrm{C}$ in a humidified $5 \% \mathrm{CO}_{2}$ incubator.

The Dearing strain of reovirus serotype 3 (Reolysin ${ }^{\circledR}$; GMP grade reovirus) was obtained from Oncolytics Biotech Inc. (Calgary, Canada).

Cell proliferation assay in reovirus-infected cell lines: Cell growth inhibition by reovirus was assessed by methylthiazole tetrazolium (MTT) assay, according to the previously published method [33]. Briefly, each cell line was seeded at an optimized number $(1,250$ cells; CIP-p, CIP-m, CHM-p and CHM-m, 2,000 cells; CMeC1, KMeC and LMeC, 2,500 cells; CTB-p, 3,000 cells; Abrams, Gracie, Moresco and CMeC2, 5,000 cells; CTB-p, CNM-m, D17, MacKinley, CMGD2 and CMGD5) in 96-well plates and was simultaneously infected or not with reovirus at MOI of $0.1,1.0,10,100$ and 1,000 PFUs per cell in triplicates. Cells were cultured for $72 \mathrm{hr}$ before MTT reagent was added into the culture for $4 \mathrm{hr}$, and the MTT assay was performed to assess cell proliferation by measuring the absorbance in the plates. All experiments were repeated at least 3 times.

Cytotoxicity assay in reovirus-infected cell lines: Each cell line was seeded at an optimized number (3,000 cells; CIP-p, CTB-p, CHM-p, CHM-m, Abrams, Gracie, Moresco, $\mathrm{CMeC} 1, \mathrm{CMeC} 2, \mathrm{KMeC}$ and $\mathrm{LMeC}$, 5,000 cells; CIP-m, CTB-m, CNM-m, D17, MacKinley, CMGD2 and CMGD5) in 96-well plates in triplicates. After $12 \mathrm{hr}$ of culture, cells were infected with mock or reovirus at MOI of 70 PFUs per cell in triplicates. At $72 \mathrm{hr}$ post-infection (hpi), live and dead cells were counted with $0.25 \%$ trypan blue. All experiments were repeated at least three times.

Viral progeny: Supernatant of the samples from the cytotoxicity assay was collected and kept at $-80^{\circ} \mathrm{C}$ until analysis. Viral progeny in the samples was measured using the $50 \%$ tissue culture infectious dose $\left(\right.$ TCID $\left._{50}\right)$ assay on L929 cells, as previously described [19] with some modifications. L929 cells were seeded at 10,000 cells in 6 replicate wells of a 96well plate. Ten serial ten-fold dilutions of the stocked virus used for infection or the culture supernatant from cytotoxicity assay were prepared and then were added into each well. After $144 \mathrm{hr}$ incubation, cytopathogenic effects (CPE) were recorded in each well, and $\mathrm{TCID}_{50}$ was calculated. Titration of the viral progeny was performed on all the supernatant from the three repeats of the cytotoxicity assay.

Reovirus infectivity: Reovirus infectivity of all the cell lines was quantified by flow cytometer. Cells were infected with reovirus at MOI of 70, and infected cells were harvested at 24 hpi (CMGD2), 48 hpi (CIP-p and CNM-m) or 72 hpi (all remaining cell lines). Cells were resuspended in PBS before being fixed and permeabilizied with BD Cytofix/Cytoperm Kit (BD Bioscience, San Diego, CA, U.S.A.). Intracellular viral protein was stained with rabbit anti-reovirus antibody, which was produced in our laboratory and was proved to be anti-reovirus specific antibody by Western blotting analysis in our previous studies $[9,10]$ and followed by incubation with anti-rabbit IgG PE antibody (Santa Cruz Biotechnology, Santa Cruz, CA, U.S.A.). Serum from healthy rabbit (GIBCO, life technologies, Tokyo, Japan) was used as an isotype control. The cells were analyzed using Cyflow Space (Partec GmbH, Munster, Germany), and the results were analyzed using FlowJo software (Tree Star, San Carlos, CA, U.S.A.).

Poly (ADP-Ribose) Polymerase (PARP) cleavage: As for PARP cleavage detection, cells were seeded at $5.0 \times 10^{5}$ cells and mock-infected or infected with reovirus at MOI of 70 before being harvested at 48 hpi. Cell pellets were lysed with NP40 lysis buffer (1\% NP40, $10 \mathrm{mM}$ Tris $\mathrm{HCl}$ $(\mathrm{pH} 7.5), 150 \mathrm{mM} \mathrm{NaCl}, 1 \mathrm{mM}$ EDTA and $1 \times$ complete mini protease inhibitor (Roche Diagnostics K.K., Tokyo, Japan)). Proteins were subjected to SDS-PAGE, followed by Western blotting. Rabbit anti-PARP antibody (NeoMarkers, Fremont, CA, U.S.A.) was used as a primary antibody, followed by secondary labeling using goat anti-rabbit IgG HRP (Zymed Laboratories, San Francisco, CA, U.S.A.). Goat anti- $\beta$-actin (Santa Cruz Biotechnology) and rabbit anti-goat IgG HRP (Bethyl Laboratories, Montgomery, TX, U.S.A.) were used as loading controls.

GST pull-down assay for Ras status: Ras activation status of all the cell lines was evaluated with GST pull-down assay as previously reported [10]. Briefly, we constructed the vector that expresses GST fused with Ras-binding domain (RBD) of Raf-1. Then, JM109 was transformed with this vector, and GST-RBD was extracted with lysis buffer. Extracted proteins from cells were mixed with gutathione- 
(A)
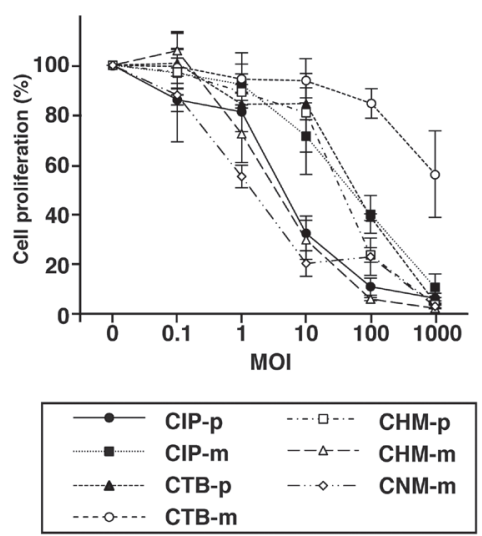

(B)
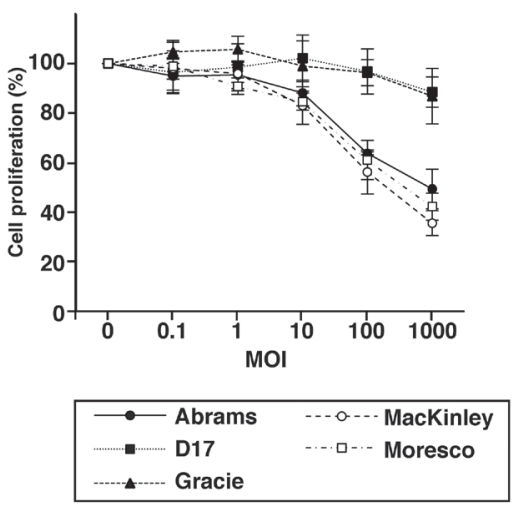

(C)
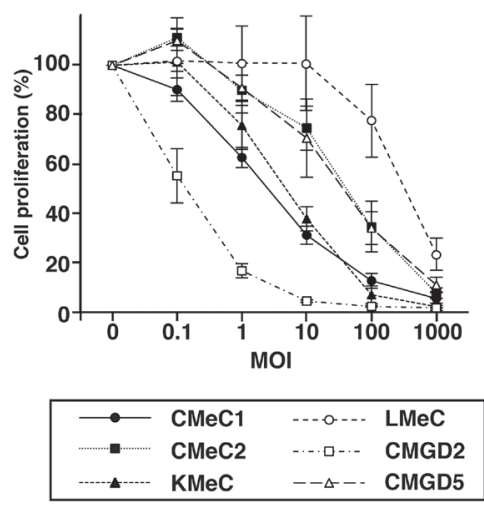

Fig. 1. Cell proliferation of canine solid tumor cell lines infected with reovirus. Canine mammary gland tumor (MGT) (A), osteosarcoma (B) and malignant melanoma (C) cell lines in triplicate wells were mock-infected or infected with reovirus at the indicated MOI. After $72 \mathrm{hr}$ post-infection (hpi), cell proliferation was quantified by MTT assay. Mean $\pm \mathrm{SD}, \mathrm{n}=3$.

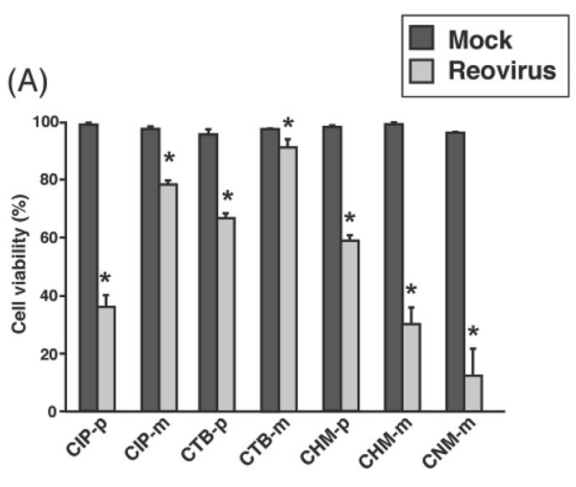

(B)

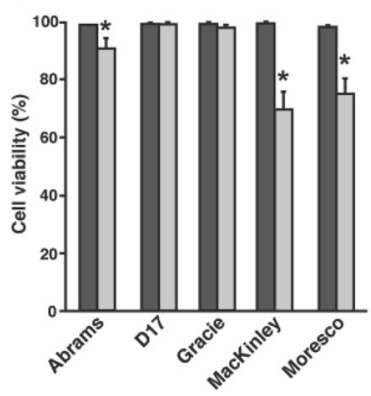

(C)

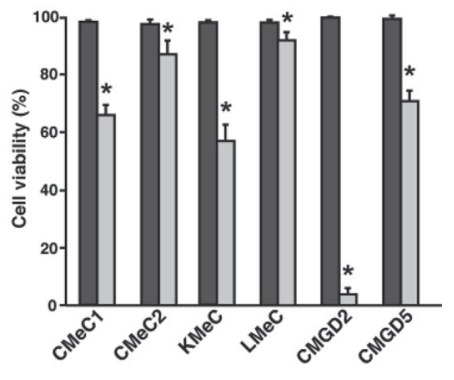

Fig. 2. Cell viability of canine solid tumor cell lines infected with reovirus. Canine MGT (A), osteosarcoma (B) and malignant melanoma (C) cell lines in triplicate wells were mock-infected or infected with reovirus at MOI 70 . After 72 hpi, cell viability was quantified by trypan blue exclusion test. Mean $\pm \mathrm{SD}, \mathrm{n}=3,{ }^{*} P<0.05$.

Sepharose 4B beads (GE Healthcare, Tokyo, Japan) for $1 \mathrm{hr}$ before washing with lysis buffer. Precipitated Ras-GTP and whole cell lysates were subjected to SDS-PAGE, followed by Western blotting. Mouse anti-pan-Ras (Calbiochem, Billerica, MA, U.S.A.) and goat anti-mouse IgG HRP (Zymed Laboratories) were used as primary and secondary antibodies, respectively.

\section{RESULTS}

Inhibition of cell proliferation by reovirus in a dosedependent manner: Firstly, examination of the susceptibility of canine solid tumors towards reovirus was conducted by infecting canine MGT, osteosarcoma and malignant melanoma cell lines with reovirus at various levels of MOI, and the inhibition of cell proliferation was assessed at $72 \mathrm{hpi}$ using MTT assay (Fig. 1). The results revealed that reovirus susceptibility varied among the cell lines.

Reovirus infection at a very high MOI of 1,000 induced tremendous cell death in all the MGT cell lines except CTBm (Fig. 1A). At a lower MOI of 10, three of the MGT cell lines showed more than $50 \%$ of inhibition of cell growth, but the other 4 cell lines were less susceptible. Generally, canine osteosarcoma cell lines showed a lower susceptibility towards reovirus as compared to MGT and malignant melanoma (Fig. 1B). The most reovirus-susceptible canine osteosarcoma cell lines, Abrams, MacKinley and Moresco, only showed approximately $50 \%$ inhibition of cell growth at MOI 1,000, and all the other cell lines seemed to be minimally susceptible to reovirus at MOI 10 .

As for canine malignant melanoma, all of the cell lines were highly susceptible to reovirus at MOI 1,000 (Fig. 1C). CMGD2 showed predominant growth inhibition at MOI as low as 1 . In contrast, $\mathrm{LMeC}$ was not susceptible to reovirus at $\mathrm{MOI}$ of lower than $100 . \mathrm{CMeCl}$ and $\mathrm{KMeC}$ were more susceptible to reovirus than $\mathrm{CMeC} 2$ and CMGD5. These results suggest that canine osteosarcoma cell lines are relatively non-susceptible to reovirus infection, but some of the 
MGT and malignant melanoma cell lines are highly susceptible to reovirus in a dose-dependent manner.

Reovirus-induced cytotoxicity: MTT assay quantifies the inhibition of cell proliferation through the reduction of total mitochondrial activity [32]. In order to examine the reovirus-induced cell death directly, all the canine solid tumor cell lines were infected with reovirus at MOI 70, which is the titer of virus used for the cytotoxicity assay in mast cell tumor cell lines and lymphoma cell lines in our previous report $[9,10]$, before trypan blue exclusion test was carried out to quantify the proportion of live and dead cells (Fig. 2). The percentages of reovirus-induced cell death assessed by the trypan blue exclusion test were consistent with the percentage of inhibition of cell proliferation by reovirus. These results indicate that the inhibition of cell proliferation by reovirus is mainly due to cell death.

Reovirus infectivity and production of reoviral progeny: Reovirus infectivity in the involved cancer cell lines was quantified by flow cytometric analysis of intracellular viral protein (Fig. 3). In CTB-m and Gracie, reovirus proteins were not detected by flow cytometry, which was consistent with the low cytotoxicity in the reovirus-infected cell lines. However, reoviral proteins were detected in the cytoplasm of some of the cell lines, such as Abrams, D17, CMeC2 and $\mathrm{LMeC}$, even though they showed low cytotoxicity after reovirus infection. Reoviral proteins were detected in all the other cell lines at various levels. Although there were some exceptions, the combination of these results shows that the detection of reoviral proteins correlates with the reovirusinduced cell death.

In order to determine if reovirus-susceptible cell lines can support viral production, we measured the amount of viral progeny by $\mathrm{TCID}_{50}$ assay. Figure 4 represents the fold increase of viral titer as compared with input viral titer in the culture supernatant. Increment of titer in culture supernatant from reovirus-infected CTB-p, CTB-m and Gracie cells showed less than 1 , which suggested that these cell lines did not produce any virus. The remaining cell lines produced a certain amount of viral progeny, which were consistent with the detection of viral proteins and reovirus-induced cell death. Among the less susceptible canine MGT cell lines, reoviral proteins were detected in CTB-p (Fig. 3), even though it did not support any production of viral progeny (Fig. 4). Canine MGT cell line CTB-m and canine osteosarcoma cell line Gracie had no increment of reovirus titer, which were consistent with no detection of reoviral proteins (Fig. 3). The canine osteosarcoma and malignant melanoma cell lines (Abrams, D17, CMeC2 and LMeC) were less susceptible to reovirus, but produced viral progeny after reovirus infection, which was consistent with detection of viral proteins by flow cytometry in all these cell lines. These results indicate that viral proteins and the production of viral progeny can be detected not only in cell lines highly susceptible, but also in some of the cell lines less susceptible to reovirus.

PARP cleavage as an indicator of apoptosis induced by reovirus: Previous reports have shown that reovirus-induced cell death was due to apoptosis $[3,4,10]$. Therefore, we investigated the cleavage of PARP, which is a hallmark of
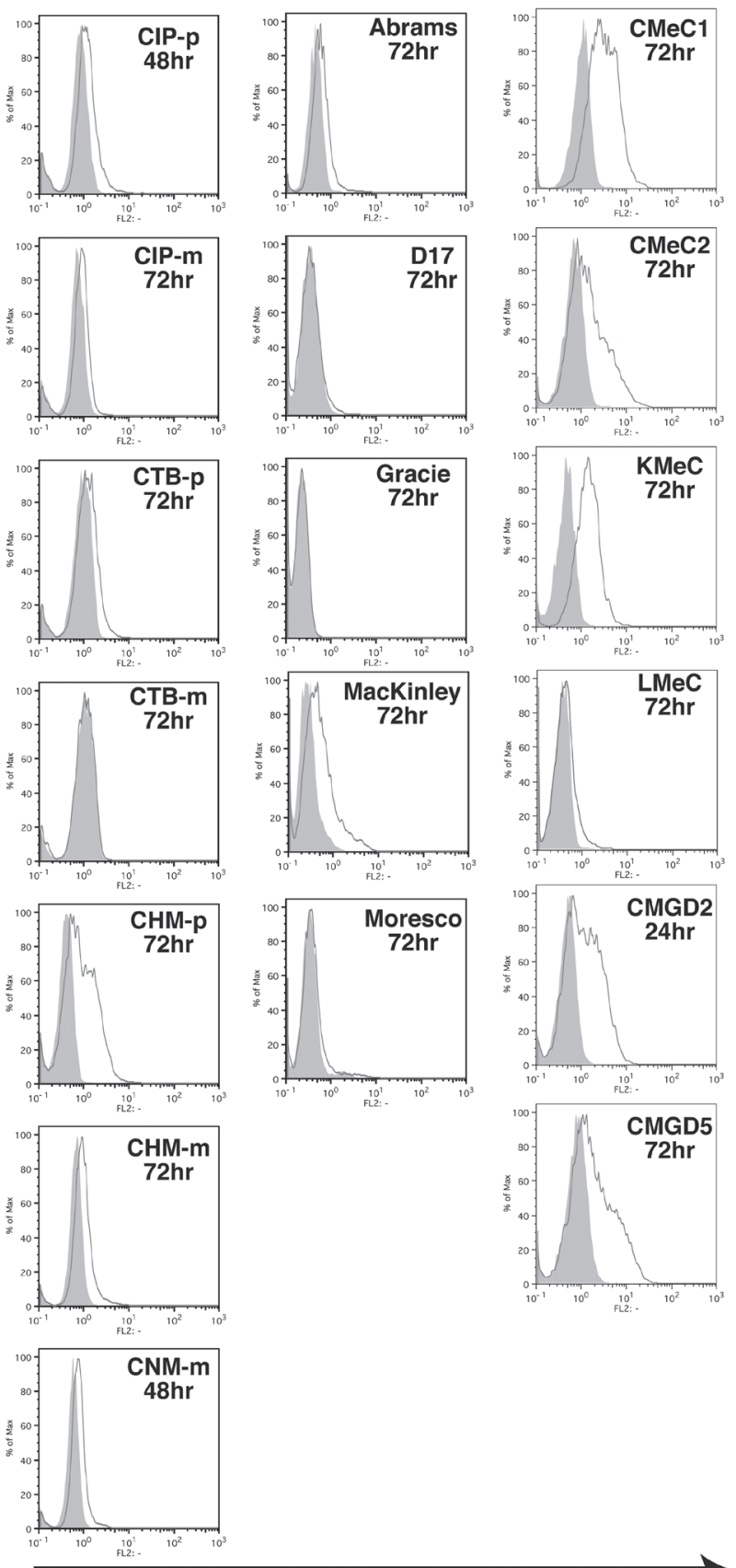

anti-reovirus

Fig. 3. Reovirus infectivity in canine solid tumor cell lines. Canine MGT, osteosarcoma and malignant melanoma cell lines were infected with reovirus at MOI 70. Reovirus-infected CMGD2 was harvested at 24 hpi. CIP-p and CNM-m were harvested at 48 hpi. All remaining cell lines were harvested at $72 \mathrm{hpi}$. Cells were stained with rabbit polyclonal anti-reovirus antibody and analyzed by flow cytometry. The shaded regions and regions below the red line in the histograms indicate staining with an isotype control and an anti-reovirus antibody, respectively. Results shown are representatives from a minimal of two repeats. 
(A)

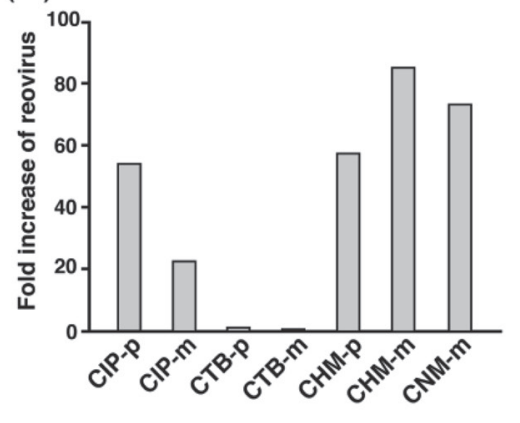

(B)

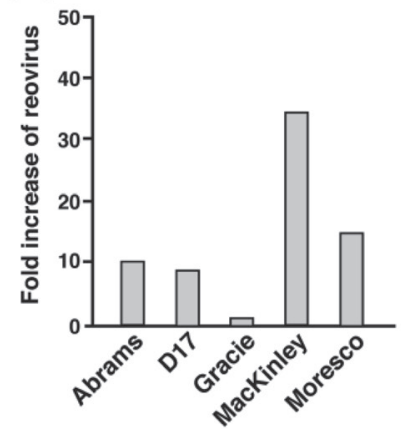

(C)

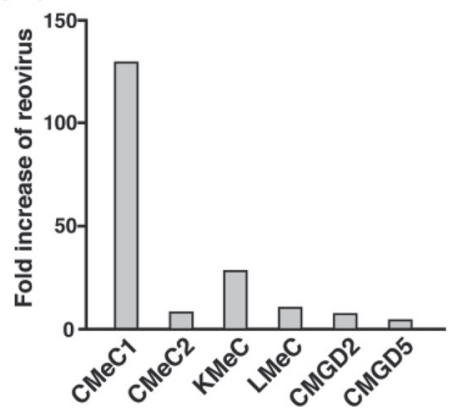

Fig. 4. Production of viral progeny in reovirus-infected canine solid tumor cell lines. Supernatant of reovirus-infected (MOI 70) canine MGT (A), osteosarcoma (B) and malignant melanoma (C) cell lines was harvested at 72 hpi, and virus titer was determined by $\mathrm{TCID}_{50}$ assay. The fold increase of reovirus represents the values calculated from the titer of progeny virus divided by the titer of input virus.
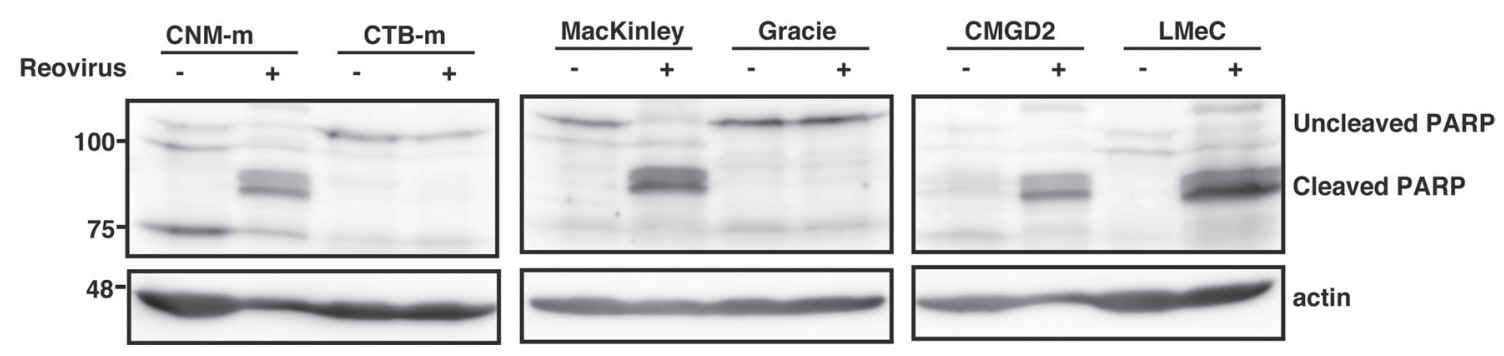

Fig. 5. Poly (ADP-Ribose) Polymerase (PARP) cleavage in selected canine solid tumor cell lines after reovirus infection. Whole cell lysates were prepared from mock-infected or reovirus-infected (MOI 70) CNM-m, CTB-m, MacKinley, Gracie, CMGD2 and $\mathrm{LMeC}$ at 48 hpi. Proteins were separated with SDS-PAGE before the cleavage of PARP was determined using Western blotting with an anti-PARP antibody. Beta-actin was used as protein loading controls. Results shown are representatives from a minimal of two repeats.

caspase-dependent apoptosis, in reovirus-infected canine MGT, osteosarcoma and malignant melanoma cell lines. The most reovirus-susceptible (CNM-m, MacKinley and CMGD2) and the least reovirus-susceptible cell lines (CTB$\mathrm{m}$, Gracie and $\mathrm{LMeC}$ ) were chosen from each of the tumor type (Fig. 5). Prominent cleaved product was detected at 48 hpi in all of the cell lines highly susceptible to reovirus. No cleaved bands were observed in reovirus-infected CTB-m and Gracie at $48 \mathrm{hpi}$, but surprisingly, cleaved PARP was detected in $\mathrm{LMeC}$, which is less susceptible to reovirus.

Lack of correlation between Ras activation status and reovirus susceptibility: The baseline GTP-loading status of Ras among the cell lines involved in this study was determined in order to investigate the involvement of Ras activation as the molecular determinant for reovirus susceptibility. In canine MGT, CHM-p and CHM-m have elevated Ras activities, while Ras activation was not detected in the other cell lines (Fig. 6A). Among the canine osteosarcoma cell lines, GTPbound Ras was highly expressed only in D17 and Gracie (Fig. 6B). In canine malignant melanoma, $\mathrm{CMeC} 1, \mathrm{CMeC} 2$ and $\mathrm{LMeC}$ have elevated Ras activities, as compared to CMGD2, CMGD5 and KMeC (Fig. 6C). The collection of these results indicates that reovirus susceptibility is not cor- related to the baseline status of Ras activation among the canine solid tumor cell lines.

\section{DISCUSSION}

We have previously reported that canine mast cell tumor is highly susceptible to reovirus infection [10], but reovirussusceptibility is less among the canine lymphoma cell lines [9]. In current human clinical studies using reovirus, solid tumors are the main targets [30]. Thus, we focused on a panel of canine tumor cell lines established from solid tumors and tested their susceptibility towards the effects of reovirus. Due to the limited availability of cell lines in the veterinary field, solid tumor cell lines established from MGT, osteosarcoma and malignant melanoma were chosen in this study (Table 1).

A total of six canine MGT cell lines, 2 canine osteosarcoma cell lines and 4 canine malignant melanoma cell lines were susceptible to reovirus, where more than $20 \%$ of the cells were killed after reovirus infection (Fig. 2). Among the 3 types of canine tumors, the osteosarcoma cell lines were relatively less susceptible to reovirus infection. On the other hand, the canine MGT and malignant melanoma cell 
(A)

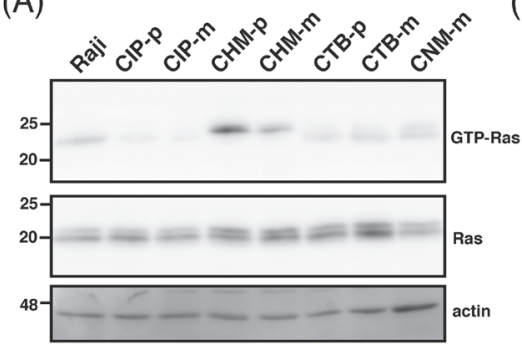

(B)

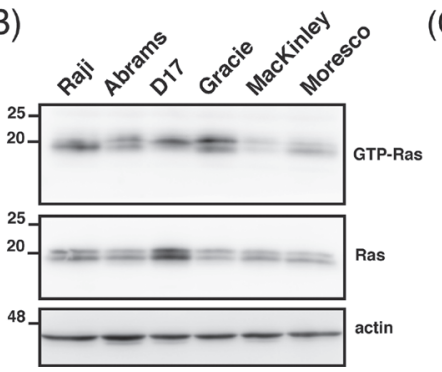

(C)

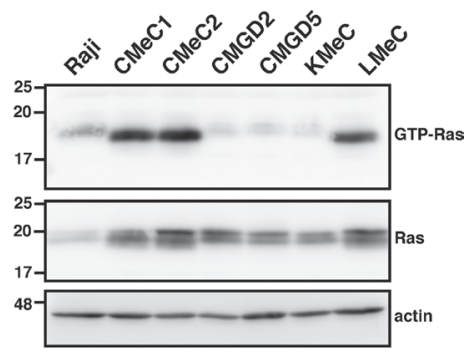

Fig. 6. Ras activation status in canine solid tumor cell lines. Ras-GTP from cell lysates of canine solid tumor cell lines was affinity-precipitated with GST-RBD protein. The affinity-precipitated products and whole cell lysates were subjected to SDSPAGE, followed by Western blotting with anti-Ras antibody, to detect Ras-GTP and total Ras, respectively. Results shown are representatives from 2 repeats.

Table 1. Summary of the effects of reovirus in canine tumor cell lines

\begin{tabular}{llcccc}
\hline Tumor type & \multicolumn{1}{c}{ Cell line } & $\begin{array}{c}\text { Reovirus } \\
\text { susceptibility }^{\text {a) }}\end{array}$ & $\begin{array}{c}\text { Viral protein } \\
\text { detection }^{\text {b) }}\end{array}$ & $\begin{array}{c}\text { Viral progeny } \\
\text { production }^{\text {c }}\end{array}$ & Ras activation \\
\hline MGT & CIPp & ++ & ++ & ++ & - \\
& CIPm & + & ++ & +++ & - \\
& CTBp & + & ++ & - & - \\
& CTBm & - & - & - & - \\
& CHMp & + & ++ & +++ & + \\
& CHMm & ++ & ++ & +++ & + \\
& CNMm & ++ & ++ & +++ & - \\
\hline OS & Abrams & - & ++ & +++ & + \\
& D17 & - & + & ++ & + \\
& Gracie & - & - & - & + \\
& Mackinley & + & ++ & +++ & - \\
& Moresco & + & + & +++ & - \\
\hline MM & CMeC1 & + & ++ & +++ & ++ \\
& CMeC2 & - & ++ & ++ & ++ \\
& KMeC & + & ++ & +++ & - \\
& LMeC & - & + & ++ & ++ \\
& CMGD2 & ++ & ++ & ++ & - \\
& CMGD5 & + & ++ & + & - \\
\hline
\end{tabular}

a)Reovirus susceptibility: - No significant difference between cell viability of mock and reovirusinfected cells; $-<20 \%$ cell death; $+20-50 \%$ cell death; $++>50 \%$ cell death. b)Viral protein detection: - No virus detection, + partly positive staining to viral protein; ++ positive staining to viral protein. c)Viral progeny production: - No increment as compared to input virus titer, $+<5 \times$ increment of virus titer; ++ 5-10× increment of virus titer; $+++>10 \times$ increment of virus titer.

lines were mostly susceptible to reovirus. Similarly, human breast cancer cell lines and malignant melanoma cell lines have been shown to be susceptible to reovirus $[6,18]$, and phase II clinical trials involving patients with breast cancer and malignant melanoma have been conducted [30]. From the results of this study, it is suggested that canine MGT and malignant melanoma are potential candidates for reovirus treatment, but not so in osteosarcoma (Table 1).

Flow cytometric analysis (Fig. 3) showed that reovirus proteins were detected in most of the cell lines except in CTB-m and Gracie, which are less susceptible to reovirus infection. Generally, the results of the production viral progeny correlated with the detection of viral proteins among the cell lines, but not in CTB-p, where viral proteins were de- tected even though there was no production of viral progeny. In normal cases, reovirus infection should occur first before virus replication and cell death. However, in our study, reovirus infection and cell death took place without virus replication in CTB-p, which is inconsistent with the other results. Our hypothesis is that CTB-p might have a low efficiency in virus replication and/ or virus release from cells, leading to the low virus titer detected in the cell culture supernatant. However, this hypothesis remains to be proven and requires further investigation.

In order to elucidate the mechanism of cell death induced by reovirus in the canine solid tumor cell lines, PARP cleavage was examined in the most reovirus-susceptible and the least reovirus-susceptible cell line from each of the tumor 
type. Our data showed that cell death in the cell lines most susceptible to reovirus, CNM-m, MacKinley and CMGD2, was accompanied with PARP cleavage, indicating that reovirus induces cell death through a caspase-dependent manner (Fig. 5), consistent with previous reports [10, 15]. Among the three cell lines that were the least susceptible to reovirus, an unexpected result was discovered, where the cleavage of PARP was detected in LMeC. In order to confirm that PARP cleavage occurs with caspase 3 activation in $\mathrm{LMeC}$, we examined the cleavage of caspase 3 with Western blotting. Our results confirmed that caspase 3 was cleaved and therefore, activated after reovirus infection took place in $\mathrm{LMeC}$ (data not shown). Hence, we came to a conclusion that there possibly exists a mechanism where apoptotic cell death can be prevented in $\mathrm{LMeC}$ even with the activation of caspase 3. However, this phenomenon remains a mystery until further investigation.

Initial studies involving reovirus as an oncolytic virus have proven that reovirus-induced cytotoxicity was observed in Ras-transformed cells but not in untransformed cells $[23,25]$. On top of that, various reports have cited that the activation of Ras signaling pathway plays a key role in enhancing reovirus disassembly, infectivity, viral replication and production viral progeny $[1,15,24]$. In this study, the results showed that profound activation of Ras was observed in CHM-p, CHM-m, D17, Gracie, CMeC1, CMeC2 and LMeC as compared to Raji, which was used as a control for Ras activation. Among the cell lines with elevated Ras activities, D17 and Gracie are the least susceptible to reovirus infection. On the other hand, CIP-p, CIP-m, CTB-p, CNM-m, MacKinley, Moresco, KMeC and CMGD2, that have low or no expression of Ras activity, are highly susceptible to reovirus. These discrepancies were also observed in some of the human cancers [21, 27, 28, 31], canine mast cell tumor [10] and canine lymphoma [9]. Indeed, we cannot completely deny that there exist other mechanisms of reovirus susceptibility besides the Ras signaling pathway. From our data, elevated Ras activity has failed to serve as a predictive marker to define the susceptibility of a tumor cell line towards reovirus. Further studies need to be performed to elucidate the alternative mechanism (s) that is involved en route to discovering the molecular determinant of reovirus susceptibility.

Reovirus serves as an attractive option in cancer therapy. Besides the influenza-like symptoms, the side effects of using reovirus as a treatment for cancers are minimal [20]. From the results of this study, it seems like reovirus as a monotherapy for cancers has limited efficacy. Different approaches using combination of reovirus with radiation or chemotherapy have been tested in human clinical trials [30]. Therefore, in order to increase the oncolytic effects of reovirus in canine MGT, osteosarcoma and malignant melanoma, further investigations on the synergistic effects of reovirus and chemotherapeutic agents in these canine cancers are currently being carried out.

In summary, this study suggested that reovirus is a potent oncolytic virotherapy in canine solid tumors, such as MGT and malignant melanoma. However, we were unable to com- pletely elucidate the mechanism of reovirus-induced cell death in these cell lines. We were also unable to identify the molecular determinant of reovirus susceptibility in canine solid tumors in this study.

ACKNOWLEDGMENTS. We sincerely thank Dr. Takayuki Nakagawa (The University of Tokyo), Dr. Douglas H. Thamm (Colorado State University) and Dr. Jaime F. Modiano (The University of Minnesota) for providing the cell lines used in this study.

\section{REFERENCES}

1. Alain, T., Kim, T. S., Lun, X., Liacini, A., Schiff, L. A., Senger, D. L. and Forsyth, P. A. 2007. Proteolytic disassembly is a critical determinant for reovirus oncolysis. Mol. Ther. 15: 1512-1521. [Medline] [CrossRef]

2. Bianco, S. R., Sun, J., Fosmire, S. P., Hance, K., Padilla, M. L., Ritt, M. G., Getzy, D. M., Duke, R. C., Withrow, S. J., Lana, S., Matthiesen, D. T., Dow, S. W., Bellgrau, D., Cutter, G. R., Helfand, S. C. and Modiano, J. F. 2003. Enhancing antimelanoma immune responses through apoptosis. Cancer Gene Ther. 10: 726-736. [Medline] [CrossRef]

3. Clarke, P., Meintzer, S. M., Wang, Y., Moffitt, L. A., Richardson-Burns, S. M., Johnson, G. L. and Tyler, K. L. 2004. JNK regulates the release of proapoptotic mitochondrial factors in reovirus-infected cells. J. Virol. 78: 13132-13138. [Medline] [CrossRef]

4. Connolly, J. L., Rodgers, S. E., Clarke, P., Ballard, D. W., Kerr, L. D., Tyler, K. L. and Dermody, T. S. 2000. Reovirus-induced apoptosis requires activation of transcription factor NF-kappaB. J. Virol. 74: 2981-2989. [Medline] [CrossRef]

5. Duncan, M. R., Stanish, S. M. and Cox, D. C. 1978. Differential sensitivity of normal and transformed human cells to reovirus infection. J. Virol. 28: 444-449. [Medline]

6. Errington, F., White, C. L., Twigger, K. R., Rose, A., Scott, K., Steele, L., Ilett, L. J., Prestwich, R., Pandha, H. S., Coffey, M., Selby, P., Vile, R., Harrington, K. J. and Melcher, A. A. 2008. Inflammatory tumour cell killing by oncolytic reovirus for the treatment of melanoma. Gene Ther. 15: 1257-1270. [Medline] [CrossRef]

7. Gobar, G. M., Case, J. T. and Kass, P. H. 1998. Program for surveillance of causes of death of dogs, using the Internet to survey small animal veterinarians. J. Am. Vet. Med. Assoc. 213: 251-256. [Medline]

8. Hashiro, G., Loh, P. C. and Yau, J. T. 1977. The preferential cytotoxicity of reovirus for certain transformed cell lines. Arch. Virol. 54: 307-315. [Medline] [CrossRef]

9. Hwang, C. C., Umeki, S., Igase, M., Coffey, M., Noguchi, S., Okuda, M. and Mizuno, T. 2014. The effects of oncolytic reovirus in canine lymphoma cell lines. Vet. Comp. Oncol. [CrossRef]. [Medline]

10. Hwang, C. C., Umeki, S., Kubo, M., Hayashi, T., Shimoda, H., Mochizuki, M., Maeda, K., Baba, K., Hiraoka, H., Coffey, M., Okuda, M. and Mizuno, T. 2013. Oncolytic reovirus in canine mast cell tumor. PLoS ONE 8: e73555. [Medline] [CrossRef]

11. Inoue, K., Ohashi, E., Kadosawa, T., Hong, S.H., Matsunaga, S., Mochizuki, M., Nishimura, R. and Sasaki, N. 2004. Establishment and characterization of four canine melanoma cell lines. $J$. Vet. Med. Sci. 66: 1437-1440. [Medline] [CrossRef]

12. Kelsey, J. L., Moore, A. S. and Glickman, L. T. 1998. Epidemiologic studies of risk factors for cancer in pet dogs. Epidemiol. 
Rev. 20: 204-217. [Medline] [CrossRef]

13. MacNeill, A. L., Moldenhauer, T., Doty, R. and Mann, T. 2012. Myxoma virus induces apoptosis in cultured feline carcinoma cells. Res. Vet. Sci. 93: 1036-1038. [Medline] [CrossRef]

14. Maeda, J., Yurkon, C. R., Fujisawa, H., Kaneko, M., Genet, S. C., Roybal, E. J., Rota, G. W., Saffer, E. R., Rose, B. J., Hanneman, W. H., Thamm, D. H. and Kato, T. A. 2012. Genomic instability and telomere fusion of canine osteosarcoma cells. PLOS ONE 7: e43355. [Medline] [CrossRef]

15. Marcato, P., Shmulevitz, M., Pan, D., Stoltz, D. and Lee, P. W. 2007. Ras transformation mediates reovirus oncolysis by enhancing virus uncoating, particle infectivity, and apoptosis-dependent release. Mol. Ther. 15: 1522-1530. [Medline] [CrossRef]

16. Merlo, D. F., Rossi, L., Pellegrino, C., Ceppi, M., Cardellino, U., Capurro, C., Ratto, A., Sambucco, P. L., Sestito, V., Tanara, G. and Bocchini, V. 2008. Cancer incidence in pet dogs: findings of the Animal Tumor Registry of Genoa, Italy. J. Vet. Intern. Med. 22: 976-984. [Medline] [CrossRef]

17. Nakagawa, T., Watanabe, M., Ohashi, E., Uyama, R., Takauji, S., Mochizuki, M., Nishimura, R., Ogawa, H., Sugano, S. and Sasaki, N. 2006. Cyclopedic protein expression analysis of cultured canine mammary gland adenocarcinoma cells from six tumours. Res. Vet. Sci. 80: 317-323. [Medline] [CrossRef]

18. Norman, K. L., Coffey, M. C., Hirasawa, K., Demetrick, D. J., Nishikawa, S. G., DiFrancesco, L. M., Strong, J. E. and Lee, P. W. K. 2002. Reovirus oncolysis of human breast cancer. Hum. Gene Ther 13: 641-652. [Medline] [CrossRef]

19. Reed, L. J. and Muench, H. 1938. A simple method of estimating fifty per cent endpoints. Am. J. Epidemiol. 27: 493-497.

20. Rosen, L., Evans, H. E. and Spickard, A. 1963. Reovirus infections in human volunteers. Am. J. Hyg. 77: 29-37. [Medline]

21. Sei, S., Mussio, J. K., Yang, Q.E., Nagashima, K., Parchment, R. E., Coffey, M. C., Shoemaker, R. H. and Tomaszewski, J. E. 2009. Synergistic antitumor activity of oncolytic reovirus and chemotherapeutic agents in non-small cell lung cancer cells. Mol. Cancer 8: 47. [Medline] [CrossRef]

22. Selb, B. and Weber, B. 1994. A study of human reovirus IgG and IgA antibodies by ELISA and western blot. J. Virol. Methods 47: 15-25. [Medline] [CrossRef]

23. Shmulevitz, M., Marcato, P. and Lee, P. W. K. 2005. Unshackling the links between reovirus oncolysis, Ras signaling, translational control and cancer. Oncogene 24: 7720-7728. [Medline] [CrossRef]

24. Shmulevitz, M., Pan, L.Z., Garant, K., Pan, D. and Lee, P. W. K. 2010. Oncogenic Ras promotes reovirus spread by suppress- ing IFN-beta production through negative regulation of RIG-I signaling. Cancer Res. 70: 4912-4921. [Medline] [CrossRef]

25. Strong, J. E., Coffey, M. C., Tang, D., Sabinin, P. and Lee, P. W. 1998. The molecular basis of viral oncolysis: usurpation of the Ras signaling pathway by reovirus. EMBO J. 17: 3351-3362. [Medline] [CrossRef]

26. Tai, J. H., Williams, J. V., Edwards, K. M., Wright, P. F., Crowe, J. E. and Dermody, T. S. 2005. Prevalence of reovirus-specific antibodies in young children in Nashville, Tennessee. $J$. Infect. Dis. 191: 1221-1224. [Medline] [CrossRef]

27. Thirukkumaran, C. M., Luider, J. M., Stewart, D. A., Cheng, T., Lupichuk, S. M., Nodwell, M. J., Russell, J. A., Auer, I. A. and Morris, D. G. 2003. Reovirus oncolysis as a novel purging strategy for autologous stem cell transplantation. Blood 102: 377-387. [Medline] [CrossRef]

28. Twigger, K., Roulstone, V., Kyula, J., Karapanagiotou, E. M., Syrigos, K. N., Morgan, R., White, C., Bhide, S., Nuovo, G., Coffey, M., Thompson, B., Jebar, A., Errington, F., Melcher, A. A., Vile, R. G., Pandha, H. S. and Harrington, K. J. 2012. Reovirus exerts potent oncolytic effects in head and neck cancer cell lines that are independent of signalling in the EGFR pathway. BMC Cancer 12: 368. [Medline]

29. Urbasic, A. S., Hynes, S., Somrak, A., Contakos, S., Rahman, M. M., Liu, J. and MacNeill, A. L. 2012. Oncolysis of canine tumor cells by myxoma virus lacking the serp2 gene. Am. J. Vet. Res. 73: 1252-1261. [Medline] [CrossRef]

30. Vacchelli, E., Eggermont, A., Sautès-Fridman, C., Galon, J., Zitvogel, L., Kroemer, G. and Galluzzi, L. 2013. Trial watch: Oncolytic viruses for cancer therapy. OncoImmunology 2: e24612. [Medline] [CrossRef]

31. van Houdt, W. J., Smakman, N., van den Wollenberg, D. J. M., Emmink, B. L., Veenendaal, L. M., van Diest, P. J., Hoeben, R. C., Borel Rinkes, I. H. M. and Kranenburg, O. 2008. Transient infection of freshly isolated human colorectal tumor cells by reovirus T3D intermediate subviral particles. Cancer Gene Ther. 15: 284-292. [Medline] [CrossRef]

32. van Meerloo, J., Kaspers, G. J. L. and Cloos, J. 2011. Cell sensitivity assays: the MTT assay. Methods Mol. Biol. 731: 237-245. [Medline] [CrossRef]

33. Yang, W. Q., Senger, D., Muzik, H., Shi, Z. Q., Johnson, D., Brasher, P. M., Rewcastle, N. B., Hamilton, M., Rutka, J., Wolff, J., Wetmore, C., Curran, T., Lee, P. W. and Forsyth, P. A. 2003. Reovirus prolongs survival and reduces the frequency of spinal and leptomeningeal metastases from medulloblastoma. Cancer Res. 63: 3162-3172. [Medline] 